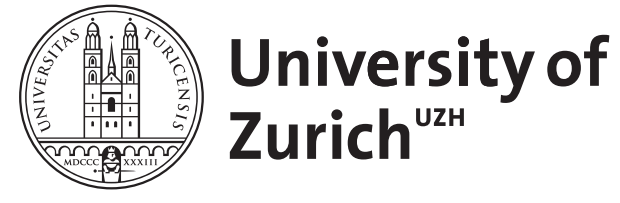

\title{
Towards a taxonomy of movement patterns
}

\author{
Dodge, S ; Weibel, Robert ; Lautenschütz, A K
}

DOI: https://doi.org/10.1057/palgrave.ivs.9500182

Posted at the Zurich Open Repository and Archive, University of Zurich ZORA URL: https://doi.org/10.5167/uzh-9627

Journal Article

Accepted Version

Originally published at:

Dodge, S; Weibel, Robert; Lautenschütz, A K (2008). Towards a taxonomy of movement patterns. Information Visualization, 7(3-4):240-252.

DOI: https://doi.org/10.1057/palgrave.ivs.9500182 


\title{
Towards a Taxonomy of Movement Patterns
}

\author{
Somayeh Dodge, Robert Weibel, Anna-Katharina Lautenschütz \\ Department of Geography, University of Zurich \\ Winterthurerstrasse 190, 8057 Zurich (Switzerland) \\ \{somayeh.dodge | robert.weibel | a-k.lautenschuetz\}@geo.uzh.ch
}

\begin{abstract}
A review of research that has been carried out on data mining and visual analysis of movement patterns suggests that there is little agreement on the relevant types of movement patterns and only few, isolated definitions of these exist. Since the research interest in this area has recently started to soar, we believe that this is a good time to approach the definition of movement patterns in a more systematic and comprehensive way. This paper intends to contribute to the development of a toolbox of data mining algorithms and visual analytic techniques for movement analysis by developing firstly a conceptual framework for movement behavior of different moving objects and secondly a comprehensive classification and review of movement patterns. We argue that this is indispensable as a basis for the development of pattern recognition and information visualization algorithms that are required to be efficient (i.e. usable on massive data sets), effective (i.e. capable of accurately detecting patterns not artifacts), and as generic as possible (i.e. potentially applicable to different types of movement data). We demonstrate the utilization of our classification by answering the question as to what extent eye tracking data can be seen as a proxy of other types of movement data. We have set up a moderated discussion platform in order to facilitate the further evolution of our proposed classification towards a consolidated taxonomy in a consensus process.
\end{abstract}

\section{Keywords}

Movement pattern, moving object, spatiotemporal data mining, visual analytics, taxonomy, behavior

\section{Introduction}

Mobility is a key element of many processes and activities, and the understanding of movement is important in many areas of science and technology such as meteorology, biology, sociology, transportation engineering, to name but a few. Hence, increasing amounts of movement tracking data and other data about the dynamics of mobile objects or agents are being collected.

By definition, moving objects are entities whose positions or geometric attributes change over time. However, in many applications the dimension of the object is not as important as its position. Hence, moving objects are considered as moving points, whose trajectories (i.e. paths through space and time) can be visualized and analyzed. By all accounts, moving objects can be categorized into two major groups of geo-referenced vs. non-georeferenced dynamic objects. In other words, some are dynamic objects that move about in geographic space and may thus be geographically referenced such as humans, animals or vehicles, while the other group includes dynamic phenomena that move in a non-geographic space, including gaze point movements in eye movement studies or particles in a bubble chamber. Each of these dynamic objects, to a varying degree, shares some similarities but also exhibits differences to the others in terms of the corresponding data structure, dynamic behavior and nature of movement.

In most cases, moving object data sets are rather large in volume and complex in the structure of movement patterns that they record. Therefore, it is necessary to develop efficient data mining algorithms and visual analytics techniques in order to extract useful and relevant information, regularities and structure from massive movement data sets. Such tools can help researchers detecting movement patterns and exploring movement behavior of different entity types.

Moving object analysis has recently become the focus of many research projects in the area of geographic information science. A review of the literature on moving object data mining and visualization highlights the importance and significant progress of these studies. However, it also suggests that there is little agreement on the relevant types of movement patterns and only few, isolated definitions of these exist. Therefore, we believe that this is a good time to approach the definition of patterns in movement data more systematically and comprehensively.

The objectives of this article are firstly to propose a conceptual framework of the elements defining the movement behavior of different moving objects and secondly the development of a comprehensive classification and definitions of movement patterns. We restrict our proposal to Moving Point Objects (MPOs), ignoring the dimension of the objects. The third objective is a call for collaboration in consolidating the proposed classification and corresponding definitions into a complete taxonomy in a moderated participatory process among domain specialists. 
There are several good reasons for a comprehensive taxonomy and accurate definitions of movement patterns. First, the design of efficient and effective data mining algorithms and objective visual analytic methods requires accurate formalization of the movement patterns and their properties. Second, most of the quoted work departs from the assumption that generic algorithms can be developed that will be suitable for different kinds of MPO data. However, this will only be possible if we know exactly the similarities but also the differences between different types of moving object data and the patterns inherent to them. Third, and related to the second point, is the argument of interoperability: Movement analysis and visualization extends across diverse disciplines and hence different people should be able to gain the same understanding of the same terms. This also applies to the 'translation' of natural language descriptions of movement patterns, as they may be collected in cognitive experiments [5]. Fourth, a classification and formalization of patterns is necessary to give guidelines for the development of visual and interactive methods that are expected to enable users to detect and explore patterns. Therefore it is a stepping-stone for the optimization of visualizations of movement data and renders pattern extraction comparable for various graphical representations and thus supports humans in their decision-making process. It also provides a starting point and interpretation guide for the visual analysis of movement data, supporting humans in identifying movement patterns. An agreement on pattern types beforehand allows an easier identification during the visual analysis process. And fifth and finally, an accurate definition of motion patterns and their constituents is also important for the evaluation of detected patterns by simulation [18].

The remainder of the paper is organized as follows: We start with a brief summary of related work on movement pattern mining and visualization. We continue by explaining the methodology used to develop our classification of movement patterns. Next, we introduce the framework of movement that forms the conceptual basis of the classification. We then introduce the classification, starting with an overview, followed by descriptions of the various patterns that we have identified. We end with a discussion and conclusions, explaining how we envision evolving and consolidating the classification into a complete taxonomy through a moderated process.

\section{Mining and visualizing Movement Patterns}

Progress in positioning and tracking technologies is giving increasing access to huge amounts of spatiotemporal movement tracking data and other data about the movement of mobile objects. Non-stop generation of space-time trajectories from different kinds of moving entities provides the possibility to discover useful and interesting information about personal and vehicular mobile behavior, to find patterns, extract their meaning, and expand our knowledge about the mobile world [23].

Data mining as a component of the KDD process is the application of specific algorithms for extracting patterns from data. In a moving object database, various data mining tasks, including exploratory data analysis, descriptive and predictive modeling, mining of association rules, and other pattern detection techniques can be applied on MPO trajectories in order to extract patterns of movement and discover spatio-temporal behavior of different types of moving objects $[8,18]$.

Generally, movement patterns include any recognizable spatial and temporal regularity or any interesting relationship in a set of movement data, whereas the proper definition (i.e. the instantiation) of "pattern interestingness" depends on the application domain. Early work on movement pattern analysis includes the simulation study of human adaptive behavior [5] and the methods developed for the spatiotemporal analysis of wild animal movements by Imfeld [13]. Recent years have witnessed almost an explosion of research activities, triggered by the advent of cheap and ubiquitous positioning and data collection technology. Selected representatives of these more recent publications include the work on the extraction of movement patterns from trajectories generated by individual users of location-based services [21, 23]; and the work on data mining of movement patterns in groups of moving objects [4, 10, 15, 19]. Furthermore, visual analytics methods for exploratory analysis of movement data have been proposed by Andrienko and Andrienko [1]. Visual analytics and information visualization serve the exploration of moving object data-in particular pattern extraction-and are built on the premise that humans are able to reason and learn more effectively in a visual setting. Information visualization research has produced a large variety of movement data representations. However there are few empirical evaluation studies to assess how useful these representations are.

The above publications document significant progress of research over the past few years. These studies usually set out with fairly accurate definitions of the patterns they are looking for-as an indispensable prerequisite to visual analysis and data mining [8] - but they tend to be restricted to a selected, narrow set of patterns. Hence, we are still facing a fundamental problem and impediment to the development of a comprehensive toolbox of movement analysis techniques: There is neither agreement on the relevant types of movement patterns nor any comprehensive and systematic definition of these. Therefore, there is a need to create a systematic classification of patterns in movement data. Andrienko and Andrienko [1] probably come 
closest to what may be termed such a comprehensive taxonomy for this purpose. However, while their proposal forms an excellent point of departure for subsequent work, the taxonomy should be better rooted in the relevant literature and the associated definitions must become more detailed and accurate.

\section{Methodology}

We started off our work by a review of the research conducted so far, including the above references as well as review articles such as [7, 12], as well as additional application-specific references. The aim of this first step was to categorize patterns of movement proposed by other researchers and to discover commonalities and differences in terminology and pattern types. Furthermore, we wanted to avoid developing redundant, conflicting terminology. The result of this first step is the classification of movement patterns presented below (Fig. 1). While this first step essentially represents a topdown approach we also approached this problem from bottom-up, decomposing movement into its constituting elements such as movement parameters, influencing factors, etc. The aim here was to develop a conceptual framework of the movement of dynamic objects that could be used to build definitions of individual movement patterns.

The following sections present the proposed conceptual framework of movement and the classification of movement patterns, followed by descriptions of the various patterns constituting the classification.

\section{Conceptual Framework of Movement}

Generally speaking, the analysis of the movements of dynamic objects has formed the basis of physical science ever since the times of Galileo and Newton, in the 17th century. Movement as a physical phenomenon is change of position. In the geographic dynamic domain, movement is defined as a shift in location of an object over time while the object maintains the same identity [26]. Movement data can be treated as a function matching pairs (entity, time moment) with position in space [1].

In order to study the movement behavior of dynamic objects, it is important to take a closer look at movement itself. In other words, it is necessary to know what exactly the parameters are that define movement, what external factors influence movement, and most importantly to understand what types of movement patterns can be composed from these primitives of movement. For this purpose, we tried to develop a conceptual framework of movement. The framework consists of the following elements discussed in the remainder of this section.

\section{Movement parameters}

We consider three major groups of movement parameters, primitive parameters, and derived parameters which we tem primary derivatives and secondary derivatives (Table 1). Primitive and derived parameters may be organized into spatial, temporal, and spatio-temporal dimensions: For instance, primitive spatial movement parameters consist of the position of the object over consecutive timestamps, and time instance (i.e. point in time) and time interval (i.e. temporal sampling rate) are primitive parameters in the temporal dimension. From these primitive parameters, several derived ones can be defined. Among the primary derivatives, distance and direction of movement are in the spatial dimension and solely a direct function of position $(x, y)$. Duration is defined as a period of time in which a movement is observed. Duration is a direct function of time and consists of one or more time intervals. Speed (i.e. rate of change of the object's position) and velocity (i.e. rate of change of position and direction) are parameters that combine both space and time dimensions, and can be derived directly from spatial position and time instances. Higherorder parameters of movement such as acceleration can be derived from primary derivatives $[5,9]$.

Of the secondary derivatives, the definition of the spatial parameters is assumed to be commonly known. For instance, spatial distribution represents a snapshot of the positions of MPOs in the database at a specific time. Sinuosity is a function of distance and refers to the degree of windingness of an object's trajectory $[20,25]$. Among the temporal parameters, temporal distribution denotes the distribution of events along the time line. Change of duration (also termed convexity in finance) denotes the rate of change of the duration between different observations of the same movement behavior (e.g. rate of change of the migration duration of a species of animal). Acceleration (i.e. rate of change of the object's speed) represents a spatio-temporal parameter derived from speed. Approaching rate is a function of speed and distance and "describes whether and how intensively a moving object approaches its destination" [20]. [9] describes some other derivatives of movement parameters, in terms of overall characteristics and dynamics of movement.

In movement analysis, it is often preferable to define movement parameters not only in an absolute sense (i.e. with respect to the external reference system) but also in a relative sense, that is, in relation to the movement of other MPOs. Relative movement parameters are particularly useful in the analysis of movements of two objects [5] or in groups of MPOs [19]. 


\begin{tabular}{|c|c|c|c|}
\hline Dimension & Primitive & $\begin{array}{c}\text { Primary } \\
\text { derivatives }\end{array}$ & $\begin{array}{l}\text { Secondary } \\
\text { derivatives }\end{array}$ \\
\hline \multirow{3}{*}{ Spatial } & \multirow{3}{*}{$\begin{array}{c}\text { Position } \\
(x, y)\end{array}$} & Distance $f(p o s n)$ & $\begin{array}{l}\text { Spatial distribu- } \\
\text { tion } f \text { (distance) }\end{array}$ \\
\hline & & Direction $f$ (posn) & $\begin{array}{l}\text { Change of direc- } \\
\text { tion } f(\text { direction })\end{array}$ \\
\hline & & $\begin{array}{c}\text { Spatial extent } \\
f(\text { posn })\end{array}$ & $\begin{array}{c}\text { Sinuosity } \\
f(\text { distance })\end{array}$ \\
\hline \multirow{2}{*}{ Temporal } & $\begin{array}{c}\text { Instance } \\
(t)\end{array}$ & Duration $f(t)$ & $\begin{array}{c}\text { Temporal } \\
\text { distribution }\end{array}$ \\
\hline & $\begin{array}{c}\text { Interval } \\
(t)\end{array}$ & Travel time $f(t)$ & $\begin{array}{l}\text { Change of dura- } \\
\text { tion } f \text { (duration) }\end{array}$ \\
\hline \multirow{2}{*}{$\begin{array}{c}\text { Spatio- } \\
\text { temporal } \\
(\mathbf{x}, \mathbf{y}, \mathbf{t})\end{array}$} & \multirow{2}{*}{ - } & Speed $f(x, y, t)$ & $\begin{array}{c}\text { Acceleration } \\
f(\text { speed })\end{array}$ \\
\hline & & Velocity $f(x, y, t)$ & Approaching rate \\
\hline
\end{tabular}

Table 1: Movement parameters

\section{Number of moving objects involved}

Obviously, moving objects show different behavior in different situations, depending on whether the MPOs are moving alone, or accompanied by other objects or in a group. [1] distinguish between 'individual movement behavior' (IMB) on the one hand and two types of movement behaviors involving multiple objects on the other hand, termed 'momentary collective behavior' (MCB, comparing movement characteristics of multiple objects at a given instance) and 'dynamic collective behavior' (DCB, looking at movement characteristics of multiple objects over a duration). While we agree with the specific role of individual movement behavior, we argue that collective movement behavior should not be approached through arbitrary slicing across the time axis (MCB) or along the time axis (DCB), as that would ignore functional relationships that exist between moving objects. By 'functional relationships' we mean behaviorally relevant relationships between members of a group that are implied by the special nature of the group (e.g. between members of a family, players in a football team, cars in a convoy). Hence, in the analysis of collective movement we differentiate between groups, which share a behaviorally relevant functional relationship (e.g. a flock of sheep, a wolf pack) and cohorts, which merely have a factor in common that may be statistically relevant, such as the similar age (called age cohort) or sex (Tab. 2).

\begin{tabular}{|l|l|l|l|}
\hline $\begin{array}{c}\text { Number of } \\
\text { MPOs }\end{array}$ & \# Obj. & Relationship & \multicolumn{1}{|c|}{ Examples } \\
\hline Individual & $\mathrm{N}=1$ & - & $\begin{array}{l}\text { Trajectory of a person over } \\
\text { a day }\end{array}$ \\
\hline Group & $\mathrm{N}>1$ & functional & $\begin{array}{l}\text { Trajectories of a flock of } \\
\text { sheep foraging on a meadow. }\end{array}$ \\
\hline Cohort & $\mathrm{N}>1$ & statistical & $\begin{array}{l}\text { Eye movement trajectories } \\
\text { of all female participants in } \\
\text { an experiment }\end{array}$ \\
\hline
\end{tabular}

Table 2: Number of objects involved in a movement

\section{Path type}

Paths of moving objects may take different forms. Some, perhaps most, moving objects travel more or less continuously, generating a continuous path (i.e. curvilinear path), exemplified by migrating elk, a pedestrian, or a car moving on a road. Such a continuous path is typically discretized into regular steps prior to computing the movement parameters [15, $24,25]$. Other moving objects will generate discontinuous paths of moves (i.e. steps) between stops $[24,25]$. Examples of these include the movement of a bee between flowers, or saccadic movements between fixations in eye movements. In this type of stop-and-go movement the stops themselves may become more important in explaining the movement than the displacements between stops, such as in eye tracking. The definition of stops is dependent on the application domain. For instance, in eye movement research fixations are identified by several methods, either by analysis of velocity, density, or duration [22].

\section{Influencing factors}

Moving objects are influenced by various factors that impact and constrain their movements. We distinguish four groups of influencing factors, one group that represent intrinsic properties of the moving object, and three groups of extrinsic factors:

- Intrinsic properties of the moving object. Each moving object has its particular intrinsic physical and behavioral movement properties, such as top speed, acceleration behavior, etc.

- Spatial constraints (networks, barriers etc.): These objects really act as a firm constraint to the movement, such as the road network constrains the movement of a car.

- Environment against which the movement takes place. Besides firm constraints, the environment is full of factors that are more or less attractive or repelling to moving objects. For instance, different vegetation influences animal movements, and the image in eye movement experiments acts as a target of attractors of varying degree.

- Influences of other agents. For instance, the movements of an animal are influenced by other members of its own or other species that induce competition, attraction, or disturbances.

Obviously, the above influencing factors vary between entity types. They are therefore non-generic and must be defined specifically for each entity type. [1] have also proposed a classification of influencing factors. We believe, however, that our classes are better defined with respect to the behavioral characteristics of movement. For instance, we devote a specific class to influences of other agents.

\section{Scale/granularity}

Depending on the influencing factors acting on a particular mobile object, the resulting movement will take place at different spatial and temporal scales. Scale issues play an important role in producing and interpreting a specific movement pattern. The spatial scale of movement-ranging from the very local scale in the order of centimeters (e.g. eye movements) to global scale (e.g. air traffic) - 
dictates the requirements on the precision and accuracy of the positioning technology used to produce movement fixes. Likewise, the temporal scale varies between very short-term and long-term behavior. Again, this imposes requirements on the temporal sampling resolution in producing movement fixes. Since the sampling may be discontinuous (i.e. may exhibit sampling gaps), since oversampling and undersampling may occur, and since many pattern analysis methods require fixes at regular time intervals, trajectories often need to be resampled prior to movement pattern detection. The temporal granularity that is generated in the resampling critically depends on the movement characteristics of the MPO in question. That is, setting the right temporal granularity requires at least some limited prior knowledge of the movement under study. Using the wrong temporal granularity may dramatically bias the resulting patterns. So, while it may still be possible to develop generic methods for data mining and visual analytics, the preprocessing of the trajectory data definitely is domainspecific. [19] provide a discussion of scale and granularity issues, quoting work by other authors as well.

\section{Classification of Movement Patterns}

In order to facilitate the detection of movement patterns, it is necessary to understand what types of patterns may exist in the data [1]. Having defined the elements of the above conceptual framework of movement, we are now in a position to attempt to develop a systematic classification of patterns in movement data. This classification and its elements should be applicable for all the common types of moving objects relevant to the geo-spatial domain, such as humans, animals, cars and eye movement data.

As explained above, our proposed classification is based on a review of the pertinent literature, in order to maximize reuse of already existing sufficiently accurate definitions, and minimize redundant, conflicting terminology. We have tried to align the definitions from the literature with the elements of our conceptual framework, and redefine and sharpen definitions where necessary. In some cases, additional pattern concepts had to be introduced to make the classification comprehensive.

In the remainder of this section, we first explain the overall organization of the classification, that is, the categories according to which the various movement patterns can be organized. This structure is also shown in Figure 1.

\section{Individual vs. group movement patterns}

In our framework, one defining element of movement patterns is whether they occur in individuals or in multiple MPOs. In Table 2, we distinguished between movements of individuals on the one hand, and groups and cohorts as representatives of multiple MPOs on the other. For the sake of defining movement patterns, however, we can neglect the type of relationship that exists between the members of a collection of moving objects (which distinguishes groups from cohorts). The type of relationship between object collections only plays a role in the interpretation of movement patterns, not in defining them. Hence, we can simply distinguish individual vs. group movement patterns.

\section{Dimension of the patterns}

Following the conceptual framework, depending on the dimension(s) used in studying the movement, patterns are categorized into three types of spatial, temporal and spatio-temporal patterns (Table 1).

\section{Generic patterns vs. behavioral patterns}

"Movement is behavior" [5]. Yet, in order to develop data mining algorithms and visual analytics methods that are as widely applicable as possible, it is helpful to try to identify patterns that may be found in any form of behavior that builds on movement. These generic patterns are usually insufficient to explain specific behavior of particular moving objects. For instance, two animal species both may exhibit periodicities in their movements, yet in one case the period may be diurnal and annual in the other. Hence, generic patterns represent the building blocks used to form higherlevel movement patterns that correspond to a particular behavior typical of a particular MPO. These patterns are then called behavioral patterns. Behavioral patterns also include movement patterns that can solely be found in certain types of MPOs (e.g. certain animal species).

\section{Primitive vs. compound patterns}

Generic movement patterns are simpler than behavioral patterns. However, even among the generic patterns, different levels of complexity can be distinguished. Hence, in our classification we distinguish between primitive and compound patterns among the generic movement patterns. Primitive patterns are the most basic forms of movement patterns, those where only a single movement parameter varies, such as incidents and constancy. In contrast, compound patterns are made up of several primitives involving complex inter-object relations, such as trend-setting, convergence, and encounter. In a similar way, [1] proposed two major types of patterns for dynamic collective behavior, called descriptive and connectional patterns, respectively. 


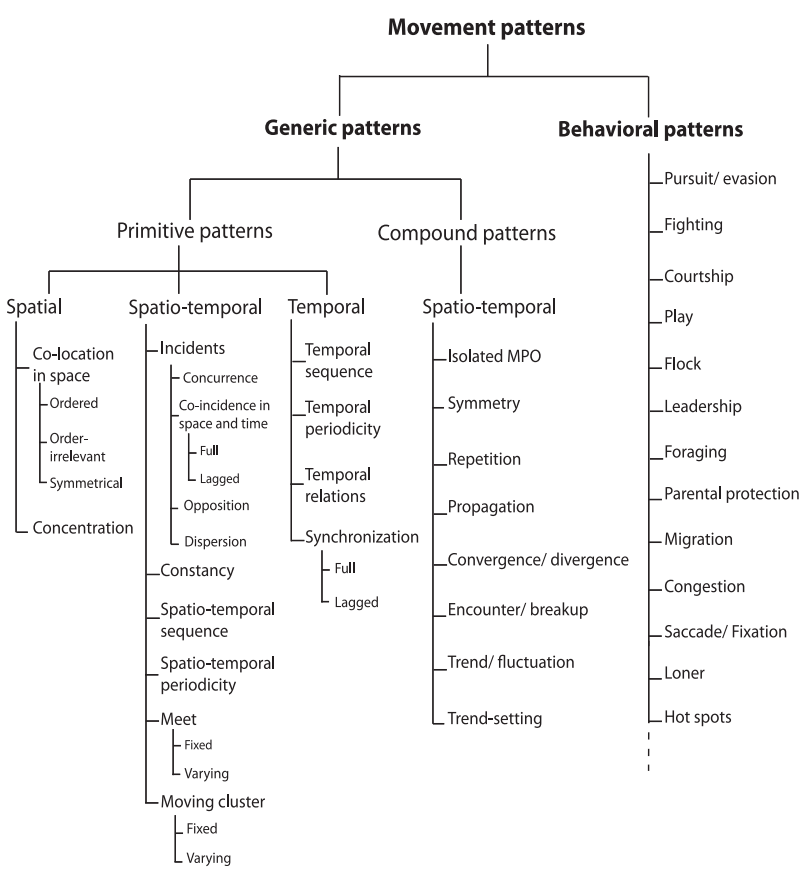

Figure 1: Classification of movement patterns

\section{Describing the Patterns}

In the following two sections we give descriptions and examples of the patterns of the proposed classification of movement patterns; first the generic patterns, then the behavioral patterns. For lack of space, the descriptions are kept short and refer to the corresponding literature. Table 3 uses the organization of the classification of Figure 1 and plots the generic movement patterns against the parameters of Table 1 that can be used to describe a particular pattern. Note that full definitions, more examples, and illustrative graphics can be found on a wiki set up by the authors [27]. The reader is cordially invited to visit this moderated website in order to discuss the proposed definitions, and contribute to the development of a consolidated, comprehensive taxonomy.

\section{Generic patterns: Primitive patterns}

Co-location in space: Occurs when the trajectories of moving objects have some positions in common [1]. There are three types of co-location in space: ordered co-location exists when the common positions are attained in the same order; unordered colocation if the common positions are attained in different orders; and symmetrical co-location when the common positions are attained similarly but in opposite orders [1]. For instance, co-location in space occurs during an eye movement experiment when different test subjects fixate on similar positions on the image; if the visiting order of fixation positions is the same, co-locations are ordered, and unordered otherwise. As another example, tourists visiting the same four places $\mathrm{A}$ to $\mathrm{C}$ in a city generate co-locations along their trajectories. If the order is from $\mathrm{A}$ to $\mathrm{C}$ in one case, and reverse in the other, then we have symmetrical co-location. Two types of co-location in space are shown in Figure 2.

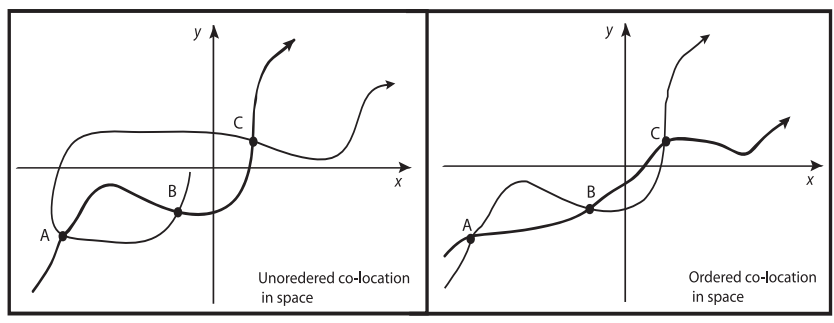

Figure 2: Co-location in space

Concentration: We dedicate a subclass of spatial patterns to spatial concentration of moving objects at a certain instance of time (e.g. A, B and C areas in Figure 3). As an example, congestion denotes a zone of high vehicle density on a transportation network. Likewise, fixations are spatially dense positions of eye movement tracks and represent concentration zones on the underlying image.

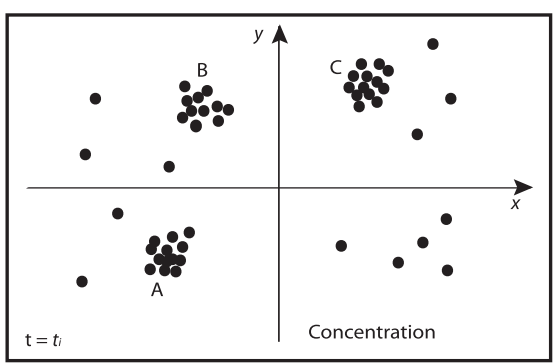

Figure 3: Concentration

Incidents: [16] introduced incidents as patterns occurring among multiple objects that can be further categorized as the following patterns:

- Concurrence: Is an incident of a set of entities showing the same values of motion attributes at a certain instant or duration. It happens when a set of objects exhibits a synchronous move- 
ment or at least similar motion parameter values over a certain duration (e.g., a flock of wild geese flying with similar motion azimuth).

- Co-incidence in space and time: [1] introduced a specific kind of incidence considering similar positions of moving objects (Figure 4). There are two variants of co-incidence patterns, full versus lagged. In the case of full co-incidence, the same positions are attained at the same time while in lagged co-incidence, it happens after a time delay. For instance, two different flocks of wild geese reach a particular pond at the same time or separated by a delay of one day.

- Opposition: A bi- or multi-polar arrangement of motion parameter values, such as the spatial splitting of a group of moving objects shown in a sudden appearance of two opposite motion directions. For instance, when flying geese are prompted to fly in opposite directions by a source of disturbance.

- Dispersion: Is the opposite of concurrence. An evident pattern in a group of MPOs that is performing a non-uniform or random motion.

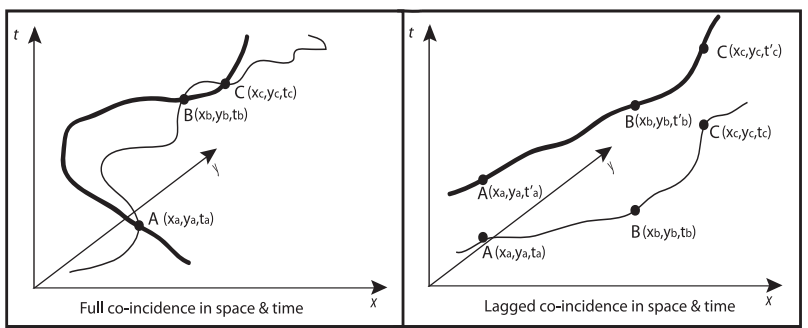

Figure 4: Co-incidence in space and time

Constancy: When the movement parameters remain the same or change insignificantly for a particular duration [1], for example, when a convoy of cars moves along a straight road, at a constant speed, speed and direction and the derived parameters remain the same. Similarly, when a flock of wild geese is heading north on the spring migration or when football players execute a forward move.

Sequence: A sequence is an ordered list of visits to a series of locations. It consists of a contiguous series of segments with a known start and end point in space and time. A spatio-temporal sequence refers to an ordered subsequence of locations with their timestamps. As an example of sequential patterns, the tendency of tourists to visit a set of places $A$ to $C$ in a particular sequence $A \rightarrow B \rightarrow C$ within specified duration could be mentioned $[6,21,3]$. Another example is the sequential order of fixations for several runs of an eye movement experiment.

Periodicity: Temporal periodic patterns indicate cyclical (e.g., yearly, weekly, or daily) phenomena [21]. [1] introduced spatio-temporal periodicity, or regular repetition as occurrence of the same patterns or pattern sequences at regularly spaced time intervals (e.g., migrating geese follow the same path every year.)
Meet: A meet pattern consists of a set of MPOs that form a stationary cluster. That is, they stay within a cylinder of a certain radius in the space-time cube; in the projection to the plane, they stay within a stationary disk of specific radius in a certain time interval (Figure 5). There are two variants of meet, fixed meet and varying meet depending on whether the objects that stay together for a certain duration are the same or change in the meeting region [11, 12]. As an example for a fixed meet pattern, we mention families of geese that gather in the fall in a specific place to form a flock. An example for a varying meet is the rental car drop-off at an airport.

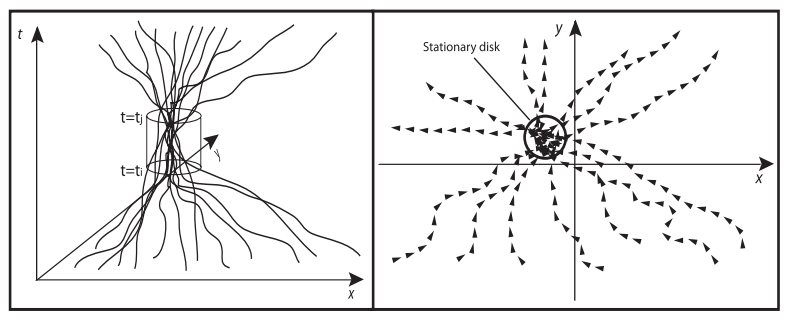

Figure 5: Meet

Moving cluster: Refers to a set of objects that stay close to each other while taking the same path for a specific duration. Nevertheless, it is not necessary that the objects participating in the pattern remain the same, but they may enter and leave, while the cluster is moving. A flock of migrating geese, a convoy of cars following the same route, and troops that move parallel on a military battlefield are different examples of moving clusters [14, 10, 7]. Based on the above definition, there are two variants of moving clusters, namely fixed moving cluster and varying moving cluster, depending on whether the participating entities stay the same or change during the observed period [11].

A moving cluster pattern is also termed a flock by some authors [4, 15]. However, we consider this a context-dependent term specifically related to biology, as in 'a flock of sheep'. Therefore, we prefer 'moving cluster' as a term for the generic form of this pattern and 'flock' as a term for the specific behavioral pattern in biology. We will discuss flock further in the section about behavioral patterns.

Temporal relations: These include any temporal relation among various events on the time axis [21]. For instance, a flock of wild geese usually stops to rest after a long continuous flight (Figure 6).

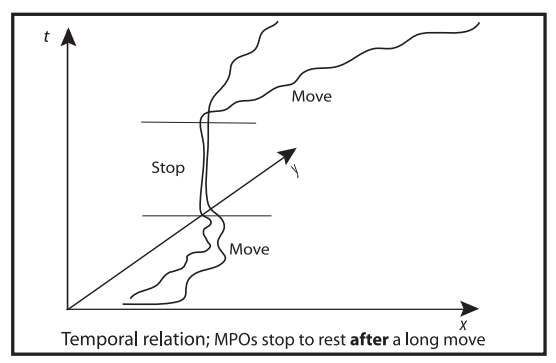

Figure 6: Temporal relations 
Synchronization in time: According to [1] there are two variants of synchronization patterns. Full synchronization happens when similar changes of movement parameters (e.g., speed, acceleration, direction, etc.) occur at the same time. In contrast, lagged synchronization happens when the changes of movement parameters occur after a time delay. E.g., forwards in football (soccer) start moving in a similar direction synchronously, when their goalkeeper kicks the ball towards the opponent's side.

\section{Generic patterns: Compound patterns}

The following patterns are all built from several primitive patterns described above, and hence termed compound patterns. Furthermore, since compound patterns consist of several primitives, they are invariably spatial and temporal.

Isolated object: Refers to an individual moving object (normally belonging to a group of MPOs) pursuing its own path, without any influence on or by the movement of other objects [16], e.g., when a goose misses the flock and travels alone.

Symmetry: Refers to sequences of patterns, where the same patterns are arranged in reverse order [1], such as wild geese heading north in the spring, and south in the fall.

Repetition: Refers to the occurrence of the same patterns or pattern sequence at different time intervals [1]. For instance, in a football match the wingers may repeatedly sprint along the sidelines or in an eye tracking experiment the test subjects may repeatedly scan the underlying image up and down.

Propagation: Propagation occurs when one object starts to show a certain movement parameter value, and little by little other objects start adopting the same pattern. By the same token, with every time step more objects are involved [16]. For instance, in the spring snow geese gradually start leaving at different times, depending on how far north they are migrating. The difference to the trend-setting pattern discussed below is that propagation always happens gradually and does not necessarily involve the influence of a 'trend-setter' object.

Convergence vs. divergence: Convergence refers to the movement of a set of objects to the same location, while the original movement direction of the involved objects does not change. In other words, the motion azimuth vectors of the objects involved will be intersecting within a specific range and within a specific duration. The objects need not arrive at exactly the same time, however. For example, several flocks of snow geese may converge toward a lake to rest. Divergence is defined as the opposite pattern of convergence and describes a group of moving objects that disperse from a common location $[10,15]$.
Encounter vs. breakup: Encounter refers to moving to and meeting at the same location. Encounter is a specific form of convergence pattern where the objects arrive at the same time (Figure 7, left). In an encounter pattern a set of MPOs have motion azimuth vectors that can be extrapolated from the current movement such that the vectors intersect within a specific range and the MPOs meet at the same time. Breakup is defined as the opposite of the encounter pattern (Figure 7, right) and describes a divergence, adding a temporal (concurrency) constraint $[10,15]$. In a football match, an encounter occurs when several players rush towards the ball and reach it at the same time. A breakup occurs when ducks flee from a pond after a gunshot is heard.

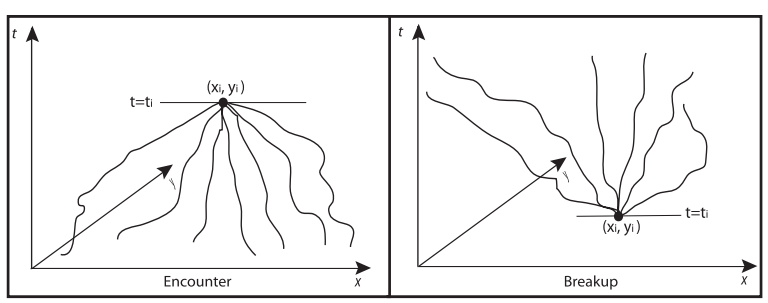

Figure 7: Encounter vs. breakup

Trend vs. fluctuation: Trend refers to consistent changes in the movement parameters of moving objects [1]. E.g., for an airplane circling in a holding pattern the rate of change of the movement direction will remain constant. Conversely, fluctuation refers to irregular changes in the movement parameters of moving objects [1], e.g., a flock of geese may change their flying formation between $\mathrm{V}$-shape, irregular V-shape, or sometimes lines.

Trend-setting: The trend-setting pattern was introduced by [15, 16]. Trend-setters are defined as objects that anticipate a certain movement pattern that is afterwards followed by a subset of the other moving objects. In another words, trend-setters are objects that influence the movement of others not necessarily in a spatial and temporal proximity $[15$, 16]. For example, in a football match, a striker executing a sudden rush towards the adversary goal acts as a trend-setter, anticipating (or triggering) a similar movement direction by the defenders and his/her own teammates). There are two variants of trend-setting, non-varying trend-setting with a fixed subset of followers and varying trend-setting [15, 16]. In the case of varying trend-setting, the subset of followers may change over the time intervals of the observation duration. Similarly to a moving cluster, in the trend-setting pattern objects move in the same direction or may have other similar movement characteristics such as same speed or acceleration $[10,2]$. The above authors use the term leadership to describe a specific kind of trendsetting pattern. Similar to the concept of a flock, we believe that leadership is a term mostly useful to describe animal and human movement behavior. 
For the same reason, we classified leadership as a type of behavioral pattern.

\section{Behavioral patterns}

As mentioned above, behavioral patterns are defined depending on the context and particular MPO type involved. Hence, for any type of moving object several behavioral patterns can be recognized that consist of generic movement patterns, yet take a particular meaning that is specific to a particular application domain. While we have tried to provide a complete list of generic movement patterns, we introduce only selected behavioral patterns for the sake of illustration. Clearly, there would be many more such patterns, as indicated in Figure 1, but we leave it to the domain specialists (e.g. behavioral ecologists, wildlife scientists) to define behaviorally relevant patterns using the generic patterns identified in this article as building blocks.

Pursuit/evasion: Evasion and pursuit belong together. Evasion refers to one animal (i.e. the prey) trying to move away and escape from a threatening, pursuing animal (i.e. the predator). They describe very high-speed movements combined with large amounts of turning and looping extending over a potentially large area of the environment [5]. Pursuit and evasion can be seen as a combination of leadership and trend-setting movements where the evader leads and affects the pursuer's movement parameters.

Fighting: Fighting is a combination of pursuit and evasion, attack and defense. Very high-speed movements are combined with large amounts of tightly intertwined turning, looping and frequent contact (where trajectories meet) in small distance between objects [5]. Fighting behavior consists of a complex combination of different generic patterns such as incidents, concurrence, repetition, colocation in space and time. If fighting occurs among a group of animals, other types of generic patterns such as convergence, divergence, encounter, breakup, and leadership might be involved.

Play: In animals, particularly juveniles, play is a form of practicing behaviors such as pursuit, evasion, fighting, or courtship. Hence, playing behavior consists of a combination of these movement behaviors, exhibiting looping, rapid dashes, and long still pauses. In play, animals repeatedly switch roles between pursuer and evader, or attacker and defender [5].

Flock: The flock pattern describes a group of animals (representing the generic pattern of a moving cluster) moving in the same direction while staying close together [10], for instance, a flock of sheep.

Leadership: The leadership pattern occurs when there is an individual that acts as the leader of a group for a specific duration. An individual can be said to be a leader if it does not follow anyone and is followed by sufficiently many other individuals at a proximate distance $[15,16]$. Leadership is a specific kind of the generic pattern trend-setting and is mostly associated with animal or human behavior. The difference to trend-setting, however, is that leadership requires spatial and temporal proximity, while in trend-setting this requirement is less stringent.

Congestion: Refers to movement with slower than usual speeds, longer trip times, and increased queuing. Extreme congestion in road traffic will lead to a traffic jam, with vehicles fully blocked for possible extended periods of time. This pattern can

\begin{tabular}{|c|c|c|c|c|c|c|c|c|c|c|c|c|c|c|}
\hline & \multirow[b]{2}{*}{ Generic patterns } & \multicolumn{2}{|c|}{$\begin{array}{c}\text { Primitive } \\
\text { param. }\end{array}$} & \multicolumn{4}{|c|}{ Primary derivatives } & \multicolumn{2}{|c|}{$\begin{array}{l}\text { Secondary } \\
\text { derivatives }\end{array}$} & \multicolumn{2}{|c|}{$\begin{array}{c}\text { Applicable } \\
\text { to }\end{array}$} & \multicolumn{3}{|c|}{ Dimension } \\
\hline & & 马ี & $\check{\Xi}$ & $\underbrace{3}_{0}$ & 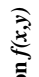 & : & 氙 & $\stackrel{\Xi}{E}$ & :ี & 焉 & $\stackrel{0}{\stackrel{0}{n}}$ & $\underset{\Delta}{\approx}$ & $\stackrel{\Xi}{E}$ & $\stackrel{8}{30}$ \\
\hline \multirow{13}{*}{ 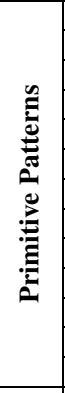 } & Co-location in space & $\mathrm{x}$ & & $\mathrm{x}$ & & & & & & & $\mathrm{x}$ & $\mathrm{x}$ & & \\
\hline & Concentration & $\mathrm{x}$ & & $\mathrm{x}$ & & & & & & $\mathrm{x}$ & $\mathrm{x}$ & $\mathrm{x}$ & & \\
\hline & Concurrence & $\mathrm{x}$ & $\mathrm{x}$ & $\mathrm{x}$ & $\mathrm{x}$ & $\mathrm{x}$ & $\mathrm{x}$ & $\mathrm{x}$ & $\mathrm{x}$ & & $\mathrm{x}$ & & & $\mathrm{x}$ \\
\hline & Co-incidence in space $\&$ time & $\mathrm{x}$ & $\mathrm{x}$ & $\mathrm{x}$ & & & & & & & $\mathrm{x}$ & & & $\mathrm{x}$ \\
\hline & Opposition & $\mathrm{x}$ & $\mathrm{x}$ & $\mathrm{x}$ & $\mathrm{x}$ & $\mathrm{x}$ & $\mathrm{x}$ & $\mathrm{x}$ & $\mathrm{x}$ & & $\mathrm{x}$ & & & $\mathrm{x}$ \\
\hline & Dispersion & $\mathrm{x}$ & $\mathrm{x}$ & $\mathrm{x}$ & $\mathrm{x}$ & $\mathrm{x}$ & $\mathrm{x}$ & $\mathrm{x}$ & $\mathrm{x}$ & & $\mathrm{x}$ & & & $\mathrm{x}$ \\
\hline & Constancy & $\mathrm{x}$ & $\mathrm{x}$ & $\mathrm{x}$ & $\mathrm{x}$ & $\mathrm{x}$ & $\mathrm{x}$ & $\mathrm{x}$ & $\mathrm{x}$ & $\mathrm{x}$ & $\mathrm{x}$ & & & $\mathrm{x}$ \\
\hline & Sequence & $\mathrm{x}$ & $\mathrm{x}$ & & & & $\mathrm{x}$ & & & $\mathrm{x}$ & $\mathrm{x}$ & & $\mathrm{x}$ & \\
\hline & Periodicity & $\mathrm{x}$ & $\mathrm{x}$ & $\mathrm{x}$ & $\mathrm{x}$ & $\mathrm{x}$ & $\mathrm{x}$ & $\mathrm{x}$ & $\mathrm{x}$ & $\mathrm{x}$ & $\mathrm{x}$ & & $\mathrm{x}$ & $\mathrm{x}$ \\
\hline & Meet & $\mathrm{x}$ & $\mathrm{x}$ & $\mathrm{x}$ & & & $\mathrm{x}$ & & & & $\mathrm{x}$ & & & $\mathrm{x}$ \\
\hline & Moving cluster & $x$ & $\mathrm{x}$ & $\mathrm{x}$ & $\mathrm{x}$ & $\mathrm{x}$ & $\mathrm{x}$ & $\mathrm{x}$ & $\mathrm{x}$ & & $x$ & & & $\mathrm{x}$ \\
\hline & Temporal relations & & $\mathrm{x}$ & & & & $\mathrm{x}$ & & & $\mathrm{x}$ & $\mathrm{x}$ & & $\mathrm{x}$ & \\
\hline & Synchronization & $\mathrm{x}$ & $x$ & $\mathrm{x}$ & $\mathrm{x}$ & $\mathrm{x}$ & $\mathrm{x}$ & $\mathrm{x}$ & $\mathrm{x}$ & & $\mathrm{x}$ & & $\mathrm{x}$ & \\
\hline \multirow{8}{*}{ 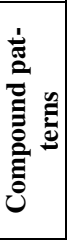 } & Isolated object & $\mathrm{x}$ & $\mathrm{x}$ & $\mathrm{x}$ & $\mathrm{x}$ & $\mathrm{x}$ & $\mathrm{x}$ & $\mathrm{x}$ & $\mathrm{x}$ & & $\mathrm{x}$ & & & $\mathrm{x}$ \\
\hline & Symmetry & $\mathrm{x}$ & $\mathrm{x}$ & $\mathrm{x}$ & $\mathrm{x}$ & $\mathrm{x}$ & $\mathrm{x}$ & $\mathrm{x}$ & $\mathrm{x}$ & & $\mathrm{x}$ & & & $\mathrm{x}$ \\
\hline & Repetition & $\mathrm{x}$ & $\mathrm{x}$ & $\mathrm{x}$ & $x$ & $\mathrm{x}$ & $\mathrm{x}$ & $\mathrm{x}$ & $\mathrm{x}$ & $\mathrm{x}$ & $\mathrm{x}$ & & & $\mathrm{x}$ \\
\hline & Propagation & $\mathrm{x}$ & $\mathrm{x}$ & $\mathrm{x}$ & $\mathrm{x}$ & $\mathrm{x}$ & $\mathrm{x}$ & $\mathrm{x}$ & $\mathrm{x}$ & & $\mathrm{x}$ & & & $\mathrm{x}$ \\
\hline & Convergence/ divergence & $\mathrm{x}$ & $\mathrm{x}$ & & $\mathrm{x}$ & & $\mathrm{x}$ & & & & $\mathrm{x}$ & & & $\mathrm{x}$ \\
\hline & Encounter/ Breakup & $x$ & $x$ & $\mathrm{x}$ & $\mathrm{x}$ & & & & & & $x$ & & & $\mathrm{x}$ \\
\hline & Trend/ Fluctuation & $\mathrm{x}$ & $\mathrm{x}$ & $\mathrm{x}$ & $\mathrm{x}$ & $\mathrm{x}$ & $\mathrm{x}$ & $\mathrm{x}$ & $\mathrm{x}$ & $\mathrm{x}$ & $\mathrm{x}$ & & & $\mathrm{x}$ \\
\hline & Trend-setting & $\mathrm{x}$ & $\mathrm{x}$ & $\mathrm{x}$ & $\mathrm{x}$ & $\mathrm{x}$ & $\mathrm{x}$ & $\mathrm{x}$ & $\mathrm{x}$ & & $\mathrm{x}$ & & & $\mathrm{x}$ \\
\hline
\end{tabular}

Table 3: Comparing the generic patterns with the elements of the conceptual framework 
be seen as a combination of meet and concurrence, along with constancy. A convoy of cars is a moving cluster that may move at slow or near-zero speed and hence lead to congestion. Similarly, traffic jams form spots of concentrations (stationary clusters).

Saccade/ fixation: In eye movement tracking studies, researchers typically analyze eye movements in terms of fixations (i.e. pauses over informative regions of interest) and saccades (rapid movements between fixations) [22]. In a spatio-temporal sense, eye movement recordings represent a combination of constancy and repetition of fast high-speed movements between fixations. In a spatial sense, they can be seen as a sequence of concentrations, as fixations represent spots of high spatial density.

\section{Mapping the classification to the conceptual framework}

Table 3 gives a summary of how the introduced conceptual framework covers the proposed classification. As mentioned before, movement patterns originate from changes in movement parameters of moving entities and depending on the dimension of characteristics involved they are categorized into spatial, temporal and spatio-temporal patterns. Table 3 shows the dependency of the patterns on variations of each movement parameter and gives an overview of patterns regarding their dimension. It also compares patterns in terms of the number of moving objects involved (individual vs. multiple MPOs). For instance, in co-incidence in space and time (Figure 4), objects have similar position at a certain instance or after a delay in time. In other words, in full co-incidence the distance between objects at a certain instance is near-zero. Consequently, the definition of the co-incidence in space and time pattern is based on three movement parameters, position, instance, and distance. Hence, this pattern is categorized as a spatio-temporal pattern (Table 3). It is also obvious that co-incidence in space and time involves multiple MPOs as it can only be defined by relations between two or more objects (Figure 4, Table 3).

\section{Discussion}

\section{Utilizing the Classification: An Example}

Moving point analysis has recently become the focus of many research projects in the area of GIScience. Many spatio-temporal data models and analytical methods have been proposed at the theoretical level, however only few of them have been implemented and applied in practice. A critical success factor for empirically based research is the availability of relevant movement data. The main problem is that data about MPOs are not easily available and accessible due to data security and privacy issues [9]. In order to overcome the problem of data scarcity, one may consider utilizing data that can act as proxies of 'physical' movement data, such as self-collected eye movement data from human subject experiments on graphic displays. Here, the main question is how similar are the movement behaviors of different MPOs. Specifically, to what extent can eye movement data be used as a proxy of other kinds of MPO data?

To answer this question, let's start with the hypothesis that the generic patterns defined above can be found in the movements of any MPO, but different MPOs will generate particular types of behavioral patterns. That is, the behavioral pattern that appears in the movement of an animal is assumed to differ from the patterns of movement of a human, and from those generated by eye movements. Here, we illustrate these similarities and differences utilizing the elements of the proposed conceptual framework and classification to compare eye movement with the whole-body movement of a human. Figures 8 and 9 show sample trajectories for these two types of movement data.

Movement parameters: All movement parameters are applicable to both eye movement and human movement data but they are different in terms of the values that they can take. For instance, eyes can move quickly in fractions of a second from one end of a picture to the other in an almost mass-less movement somewhat akin of 'teleporting', while the acceleration of human whole-body motion is dictated by greater mass and inertia.

Number of objects involved: Eye movement trajectories can be analyzed for individual test subjects or as cohorts with other subjects, which have a factor in common (e.g., the same sex, age, etc.). Human whole-body movement can be considered for an individual, in a group (e.g. family), or cohorts.

Path type: Owing to the different physics underlying the movement process, eye movements are best represented as discontinuous paths with stop-andgo sequences, while human whole-body movement best matches the continuous path model, depending on the spatial and temporal study scale.

Influencing factors: Completely different, domainspecific factors affect eye movement and human whole-body movement. For eye movement e.g. the content of the test image, vs. physical obstacles, topography, and other persons in human movement.

Scale/granularity: Eye movement and human motion are very different with respect to the spatial and temporal scales involved. The spatial scale of eye movement tracking in laboratory experiments is in the order of centimeters; for humans it varies between several meters and global scale. Similarly, the temporal scale of movement differs from fractions of seconds for eyes, to minutes and days for humans. 
Movement patterns: There are some similarities and differences between patterns generated from eye movement or human motion. For example, overlaid eye movement data from all subjects participating in an eye tracking experiment might (coincidentally) show a pattern similar to a moving cluster. Similarly, a group of people taking part in guided tour to an exhibition can be conceived as a moving cluster too. However, the group of exhibition visitors forms a functional group (somewhat akin to a 'flock'), while the collective movement behavior of the eyes of multiple test persons does not share a functional relationship, and would be simply the result of an 'overlay' of individual behaviors.

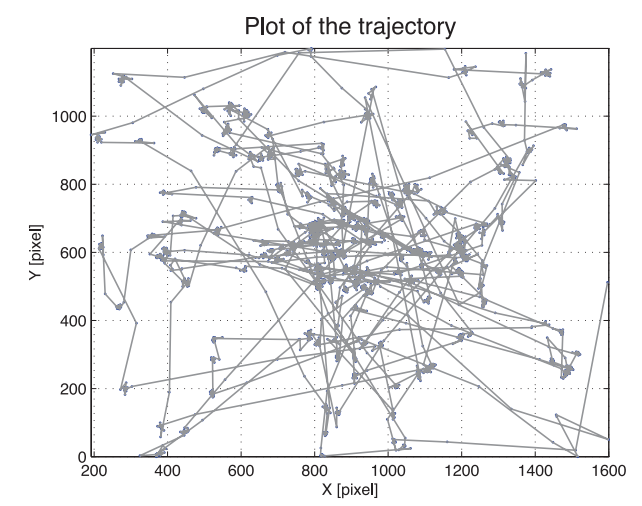

Figure 8: Sample trajectory of eye movement (data courtesy of Arzu Coltekin, The University of Zurich)

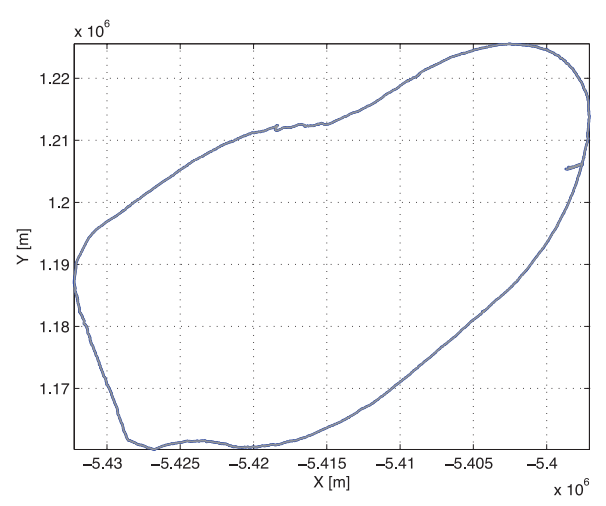

Figure 9: Sample trajectory of human movement (pedestrian) (data courtesy of www.openstreetmap.com)

It is also conceivable that motion data of eyes and humans can be made comparable by stretching and compressing the spatial and temporal scales. Thus, for instance, the displacements of a person recorded over a day (between home, office, restaurant, sports club etc.) could be made to resemble to some extent the saccadic movements of eyes between fixations. Such transformations could help in exploiting the full potential of generic pattern detection procedures. However, clearly, the interpretation of the movement behavior (which is usually the ultimate goal of the analysis) must take place at the original scale(s) and context dictated by the application domain - and hence, the existence of an inverse transformation of scales absolutely indispensable.
So, we can reach a first conclusion: There are some commonalities between eye and human whole-body movements on the level of generic movement patterns - but only as far as they stay within the bounds of the different motion physics and scales involved. In terms of behavioral movement patterns, the two types of movement are very different.

The above discussion was purely conceptual. We are currently conducting empirical research to test our hypothesis in a more systematic way considering computational and visual data mining methods in pattern detection on the one hand, and perception and cognition experiments on the other hand.

\section{Conclusions: A Call for Collaboration}

With this paper we aim to contribute to the development of a comprehensive toolbox of data mining algorithms and visual analytics methods for movement analysis. We argue that thorough definitions for a commonly agreed set of movement patterns are indispensable as a basis for the development of pattern recognition algorithms that are required to be efficient, effective, and as generic as possible. We also believe that such work is needed to support the information visualization community in developing objective visual analytics methodologies. The generic patterns identified in our classification allow a domain-independent visualization of movement. It is therefore usable for movement researchers from various disciplines, because all generic patterns should be applicable to all moving datasets at all spatio-temporal resolutions. Bees for instance generate a cyclic pattern where they return to their hive, just like wolves. Both animals produce the same kind of pattern and the pattern can be visualized accordingly although these patterns appear on different spatio-temporal resolutions. By classifying generic patterns, we also gain an advantage in identifying and exploring behavioral patterns as these consist of the generic pattern types.

This paper has contributed a conceptual framework of movement and a classification of movement patterns. The descriptions and definitions associated with the classification had to be kept short in this paper. We have therefore set up a wiki [27] for two reasons. First, the wiki hosts more background information and more detailed definitions. And even more importantly, we see this as a mechanism to facilitate the further development and consolidation of the proposed classification and pattern definitions into a complete taxonomy of movement patterns. We are convinced that generic pattern definitions must be based on the consensus of domain experts from various disciplines involved in the analysis of movement. Hence, a moderated revision process seems to have the best potential for developing a taxonomy of movement patterns that are useful on a broad range. 


\section{References}

1. Andrienko, N. \& Andrienko, G. Designing Visual Analytics Methods for Massive Collection of Movement Data. Cartographica 2007, 42(2): 117138.

2. Andersson, M., Gudmundsson, J., Laube, P., \& Wolle, T. Reporting leadership patterns among trajectories; SAC '07: Proceedings of the 2007 ACM symposium on Applied computing, ACM 2007, 3-7.

3. Agrawal, R. \& Srikant, R. Mining Sequential Patterns Eleventh International Conference on Data Engineering 1995, IEEE Computer Society, 3.

4. Benkert, M., Gudmundsson, J., Hübner, F. \& Wolle, T. Reporting Flock Patterns. Lecture Notes in Computer Science, Springer; 2006. 4168: 660-671.

5. Blythe, P.W., Miller, G.F. \& Todd, P.M. Human Simulation of Adaptive Behavior: Interactive Studies of Pursuit, Evasion, Courtship, Fighting, and Play. Proceedings of the Fourth International Conference on Simulation of Adaptive Behavior, 1996. Cambridge, MA: MIT Press/Bradford Books. 13-22.

6. Cao, H.; Mamoulis, N. \& Cheung, D. Mining frequent spatio-temporal sequential patterns. In: Fifth IEEE Intl. Conference on Data Mining, 2005, 8.

7. Erwig, M. Toward Spatiotemporal Patterns. In: Caluwe, Rita de, Tré, Guy de \& Bordogna, Gloria (eds.): Spatio-Temporal Databases - Flexible Querying and Reasoning. Springer, 2004, 29-54.

8. Fayyad, U., Piatetsky-Shapiro, G. \& Smyth, P. From Data Mining to Knowledge Discovery in Databases. AI Magazine, 1996, 17(3): 37-54.

9. Giannotti, F. \& Pedreschi, D. (eds.) Mobility, Data Mining and Privacy - Geographic Knowledge Discovery. Springer, 2007

10. Gudmundsson, J., van Kreveld, M.J. \& Speckmann, B. Efficient Detection of Motion Patterns in Spatiotemporal Data Sets. ACM GIS 2004, 250-257.

11. Gudmundsson, J. \& van Kreveld, M. Computing Longest Duration Flocks in Trajectory Data. In: Proceedings of the 14th annual ACM international symposium on Advances in geographic information systems 2006 (GIS '06), $35-42$.

12. Gudmundsson, J., Laube, P. \& Wolle, T. Movement Patterns in Spatio-Temporal Data. In: Shekhar, S. \& Xiong, H. (eds.). Encyclopedia of GIS, Springer, 2008.

13. Imfeld, S. Time, Points and Space - Towards a Better Analysis of Wildlife Data. PhD Thesis, Department of Geography, University of Zurich, 2000.

14. Kalnis P., Mamoulis N. and Bakiras S. On discovering moving clusters in spatio-temporal data. In: Proc. 9th International Symposium on Spatial and Temporal Databases 2005 (SSTD '05), 364-381.

15. Laube, P. Analysing Point Motion - SpatioTemporal Data Mining of Geospatial Lifelines. PhD Thesis, Dept. of Geography, Univ. of Zurich, 2005.

16. Laube, P., Imfeld, S. Analyzing relative motion within groups of trackable moving point ob jects. In GIScience 2002, Lecture Notes in Computer Science, Springer, 2478, 142-144.

17. Laube P., van Kreveld M., and Imfeld S. Finding REMO--Detecting relative motion patterns in geospatial lifelines, in Developments in Spatial Data Handling, (Proc. Spatial Data Handling 2004), 2004, Springer, 201-214.

18. Laube, P. \& Purves, R. An Approach to Evaluating Motion Pattern Detection Techniques in Spatio- temporal Data. Computers, Environment and Urban Systems, 2006, 30: 347-374.

19. Laube, P., Imfeld, S. \& Weibel R. Discovering Relative Motion Patterns in Groups of Moving Point Objects. International Journal of Geographical Information Science, 2005, 19(6): 639-668.

20. Laube, P., Dennis, T., Forer, P. \& Walker, M. Movement beyond the snapshot - Dynamic analysis of geospatial lifelines Computers, Journal of Environment and Urban Systems, 2007, 31: 481-501

21. Lee, J.W., Paek, O.H. \& Ryu, K.H. Temporal Moving Pattern Mining for Location-based Services. Journal of Systems and Software, 2004, 73: 481-490

22. Salvucci, D.D., \& Goldberg, J. H. Identifying fixations and saccades in eye-tracking protocols. Proc. of the Eye Tracking Research and Applications Symposium 2000, New York: ACM Press, 71-78.

23. Smyth, C.S. Mining mobile trajectories. In: Miller, H.J. \& Han, J. (eds.): Geographic Data Mining and Knowledge Discovery, Taylor \& Francis, 2001, 337361.

24. Turchin, P. Quantitative Analysis of Movement: Measuring and Modelling Population Redistribution in Animals and Plants. Sunderland, Massachusetts: Sinauer Publishers, 1998.

25. Benhamou, S.; How to reliably estimate the tortuosity of an animal's path: Straightness, sinuosity, or fractal dimension? Journal of Theoretical Biology, 2004, 229: 209-220.

26. Yuan, M. \& K. Stewart Hornsby, Computation and visualization for the understanding dynamics of geographic domains: A Research Agenda, CRC Press, New York, NY, 2007.

27. URL of the Movement Pattern Wiki: http://movementpatterns.pbwiki.com/Patterns-ofMovement 